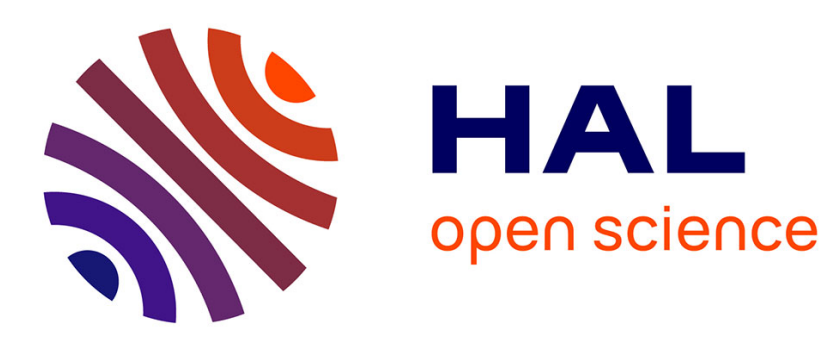

\title{
Déformation plastique d'alliages c.f.c. à teneur moyenne ou élevée en soluté
}

\author{
N. Clément, A. Coujou, P. Coulomb, J.L. Martin
}

\section{To cite this version:}

N. Clément, A. Coujou, P. Coulomb, J.L. Martin. Déformation plastique d'alliages c.f.c. à teneur moyenne ou élevée en soluté. Revue de Physique Appliquée, 1980, 15 (4), pp.831-834. 10.1051/rphysap:01980001504083100 . jpa-00244791

\section{HAL Id: jpa-00244791 https://hal.science/jpa-00244791}

Submitted on 1 Jan 1980

HAL is a multi-disciplinary open access archive for the deposit and dissemination of scientific research documents, whether they are published or not. The documents may come from teaching and research institutions in France or abroad, or from public or private research centers.
L'archive ouverte pluridisciplinaire HAL, est destinée au dépôt et à la diffusion de documents scientifiques de niveau recherche, publiés ou non, émanant des établissements d'enseignement et de recherche français ou étrangers, des laboratoires publics ou privés. 


\title{
Déformation plastique d'alliages c.f.c. à teneur moyenne ou élevée en soluté
}

\author{
N. Clément $\left({ }^{*}\right)$, A. Coujou (*), P. Coulomb $\left({ }^{*}\right)$ et J. L. Martin $\left({ }^{* *}\right)$ \\ (*) Laboratoire de Physique Electronique - Etude du Métal, U.P.S. Toulouse, France \\ $\left(^{* *}\right)$ Laboratoire d'Optique Electronique du C.N.R.S., Toulouse, France
}

(Reçu le 19 octobre 1979, révisé le 10 décembre 1979, accepté le 12 décembre 1979)

\begin{abstract}
Résumé. - Deux exemples de déformation in situ dans un microscope à très haute tension d'alliages à teneur moyenne ou élevée en soluté $(\mathrm{Ni}-33 \% \mathrm{Cr}$ et $\mathrm{Cu}-6,5 \% \mathrm{Si})$ montrent l'hétérogénéité de la déformation, les mouvements collectifs de défauts et les aspects spécifiques à des alliages présentant respectivement de l'ordre à courte distance et de l'effet Suzuki.
\end{abstract}

\begin{abstract}
Two examples of in-situ deformation in a HVEM, of alloys with an intermediate or high solute content (Ni-33 pct $\mathrm{Cr}$ and $\mathrm{Cu}-6.5$ pct $\mathrm{Si}$ ) show the inhomogeneity of deformation, the collective movements of defects and specific effects for alloys presenting respectively short range order or Suzuki effect.
\end{abstract}

Si la déformation plastique d'un métal pur de structure cubique à faces centrées (c.f.c.) s'analyse bien à partir des paramètres $\left({ }^{1}\right) \gamma / G b$ et $T$ et si le durcissement d'un alliage c.f.c. dilué est assez bien compris [1], le problème du durcissement et des modes de déformation des alliages c.f.c. à teneur moyenne ou élevée en soluté a rarement été traité dans son ensemble. Les mécanismes d'interaction d'une dislocation avec les éléments d'alliage sont connus $[2,3]$ : Effet de taille (effet Cottrell), ordre à courte distance, interaction chimique (effet Suzuki), effet de module; leur rôle dans la déformation plastique l'est moins, car il dépend de leur importance relative, mais aussi de la répartition tridimensionnelle des défauts et de leur comportement dynamique.

Nous avons étudié la déformation macroscopique (essai de traction) et microscopique (in situ en microscopie électronique à haute tension) de deux alliages de substitution typiques : $\mathrm{Ni}-33 \% \mathrm{Cr}$ en atomes $\left(\gamma / G b \simeq 3,5 \times 10^{-3} ; \gamma \simeq 75 \mathrm{~mJ} / \mathrm{m}^{2}[4]\right)$ présente une énergie de défaut moyenne et une tendance à l'ordre à courte distance; $\mathrm{Cu}-6,5 \% \mathrm{Si}$ en atomes $\left(\gamma / G b \simeq 0,75 \times 10^{-3} ; \gamma \simeq 10 \mathrm{~mJ} / \mathrm{m}^{2}\right.$ [5]) a une énergie de défaut faible et présente toutes les caractéristiques pour que l'effet Suzuki soit important.

1. Nickel-chrome. - Les alliages fortement concentrés présentent rarement une répartition aléatoire des

( $\left.{ }^{1}\right) \gamma=$ énergie spécifique de défaut d'empilement intrinsèque. $G=$ module de cisaillement $; b=$ vecteur de Burgers; $T=$ température. atomes, ils manifestent une tendance à l'ordre à courte distance, ou au contraire à la précipitation. $\mathrm{Au}$ voisinage de la stoechiométrie $\mathrm{Ni}_{2} \mathrm{Cr}$ et pour des températures un peu inférieures à la température de Kurnakov $T_{\mathrm{K}}$, on obtient par un traitement prolongé un alliage ordonné à longue distance. Pour une température un peu supérieure à $T_{\mathrm{K}}$, il existe un ordre à courte distance que la diffusion des neutrons produits par un réacteur à haut flux (à l'Institut Laue Langevin) et l'analyse de l'intensité neutronique diffusée entre les pics de Bragg permettent de mettre en évidence et d'évaluer; le phénomène est particulièrement net après un recuit de $250 \mathrm{~h}$ à $600^{\circ} \mathrm{C}$ [6]. Une analyse antérieure des courbes de traction et des paramètres d'activation des alliages nickel-chrome ordonnés à courte distance, avait montré un comportement particulier au-dessus de $20 \%$ de chrome : volume d'activation inférieur à $100 \mathrm{~b}^{3}$ et indépendant de la déformation [7]. Pour préciser les mécanismes microscopiques en jeu dans ces alliages désordonnés d'une part, ordonnés à courte distance d'autre part $\left(250 \mathrm{~h}\right.$ à $\left.600^{\circ} \mathrm{C}\right)$, nous avons fait une étude de déformation in situ dans un microscope à très haute tension, les électrons étant accélérés sous $800 \mathrm{kV}$. Les clichés $1 a$ et $b$ montrent la même plage d'une lame de $1 \mu \mathrm{m}$ d'épaisseur, après recuit, puis après une faible déformation $(2$ à $3 \%)$. On observe que le mode de déformation prédominant est le glissement coplanaire d'un très grand nombre de dislocations, formant des empilements figure $1 b[3$, $4,5,6,7]$. Une seule source peut émettre un millier 

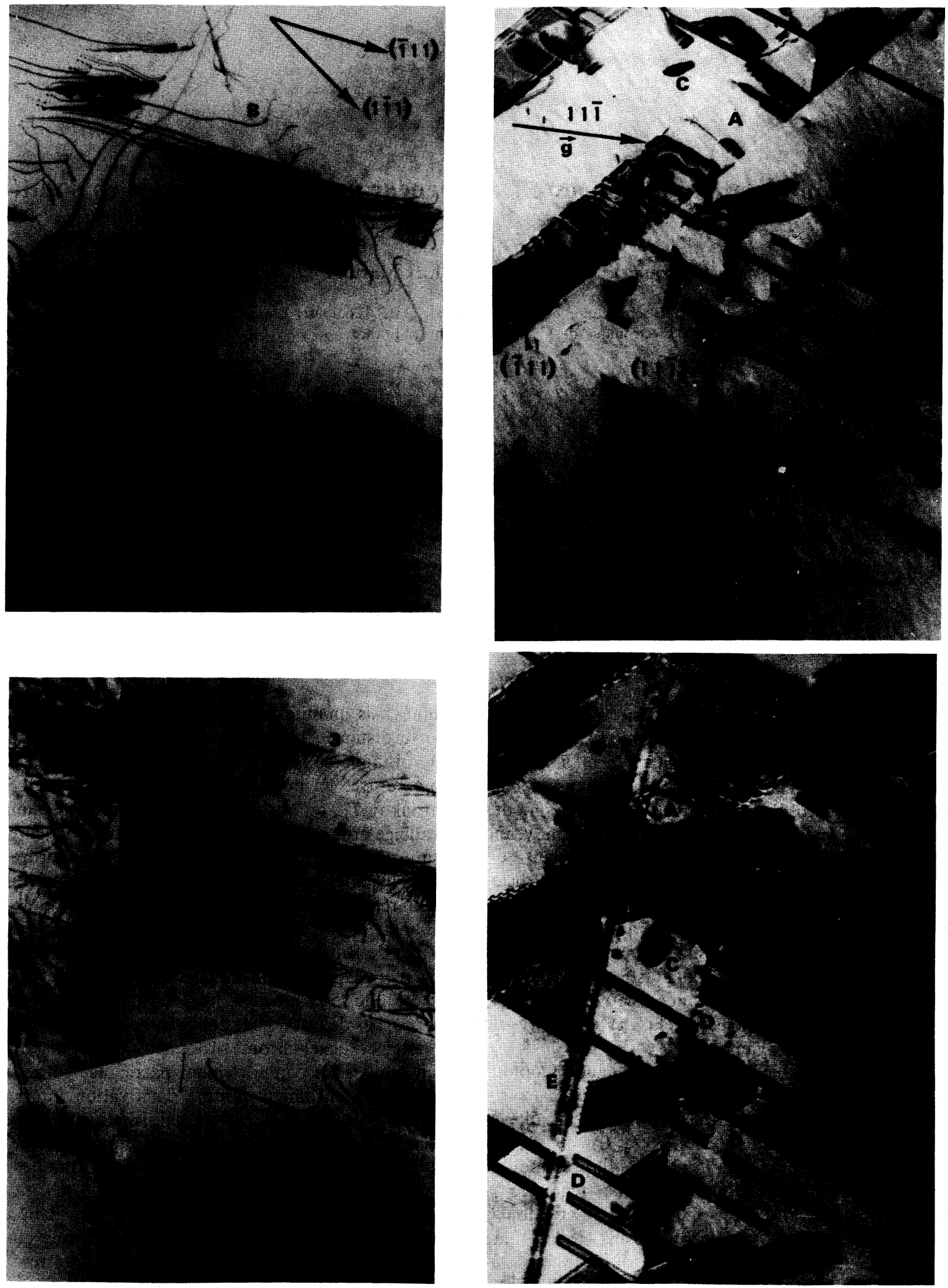

Fig. 1. - Alliage nickel- $33 \%$ chrome : $a$ ) état initial ; $b$ ) après une légère déformation $(T=300 \mathrm{~K}$, microscopie électronique à $800 \mathrm{kV}$ ).

[Nickel-33 atom pct chromium alloy : $a$ ) initial state ; $b$ ) after a slight deformation $(T=300 \mathrm{~K}, 800 \mathrm{keV}$ electron microscopy).]

Fig. 2. - Alliage cuivre- $6,5 \%$ silicium : $a$ ) état initial ; $b$ ) après une légère déformation $(T=500 \mathrm{~K}$, microscopie électronique à $800 \mathrm{kV})$.

[Copper-6.5 atom pct silicon alloy : $a$ ) initial state; $b$ ) after a slight deformation ( $T=500 \mathrm{~K}, 800 \mathrm{keV}$ electron microscopy).] 
de dislocations sur un seul plan; elles présentent des fluctuations de vitesse (autour de $10 \mu \mathrm{m} / \mathrm{s}$ ), les dislocations de tête étant mises en mouvement par l'arrivée d'un groupe mobile de dislocations.

Cette déformation très hétérogène de la lame conduit à des concentrations locales de contraintes. Une dislocation isolée ne bouge pas, sauf si elle est très proche d'un plan de glissement actif; le glissement dévié est peu fréquent ; certains empilements s'arrêtent sans qu'il y ait d'obstacle apparent à leur tête (3 sur la figure $1 b$ ). Ces observations montrent un frottement important sur les dislocations : ces forces de frottement ont été estimées à partir de la distance de séparation des dislocations successives le long des empilements observés ; elles sont fortes sur la dizaine de dislocations de tête : de l'ordre de la limite élastique pour les alliages désordonnés; elles fluctuent ensuite autour de zéro [8]. Ce comportement peut s'expliquer par la présence d'un ordre à courte distance qui est fortement diminué par le passage des dislocations de tête.

2. Cuivre-silicium. - Cet alliage a une faible énergie de défaut $\gamma$ et une grande sensibilité de $\gamma$ à la concentration $\left(\partial \gamma / \partial c \sim-300 \mathrm{~mJ} / \mathrm{m}^{2}\right)$. Dans ces conditions les rubans de défaut attirent le soluté, s'étalent et restent fixés. L'épinglage est mis en évidence à partir de $-20^{\circ} \mathrm{C}$ par des expériences de vieillissement statique qui donnent une idée de la cinétique de remplissage des défauts [9]. Au-dessus de $50^{\circ} \mathrm{C}$, l'effet Portevin-Le Chatelier est la manifestation du vieillissement dynamique, lié à l'ancrage des dislocations mobiles. Les clichés obtenus avec des électrons accélérés sous $800 \mathrm{kV}$, que nous montrons sur la figure 2 correspondent à une traction à $200^{\circ} \mathrm{C}$, température où l'on observe dès la limite élastique des discontinuités très serrées $(\Delta \varepsilon \simeq 0,01 \%)$ dues à l'effet Portevin-Le Chatelier, pour une large gamme de vitesses de déformation. L'alliage aminci (Fig. $2 a$ ) présente des dislocations fortement dissociées. Le contraste de l'empilement $\mathrm{A}$ indique qu'il est constitué par une succession de dislocations partielles glissant sur des plans superposés et constituant " une micromacle ». Après la déformation plastique (Fig. 2b), on voit que toutes les dislocations partielles ont bougé, par déplacement individuel, ou par mouvement collectif. Les déplacements individuels comprennent un étalement des dislocations dissociées (B) ou des boucles (C) autour de leur position initiale. Cet étalement doit provenir d'une ségrégation de soluté qui entraîne une diminution locale de $\gamma$ (un tel étale- ment n'est pas visible à la température ambiante où la diffusion est beaucoup plus lente). On observe aussi, en $D$ par exemple, le départ d'une dislocation partielle qui laisse derrière elle un défaut d'empilement. Cette partielle est souvent issue de l'intersection de deux micromacles comme l'avaient prévu Mahajan et Chin [11], et plus rarement d'une dislocation dissociée conformément au modèle de Hirth [12]. Dans son déplacement la partielle peut être arrêtée soit par une micromacle, soit par un autre défaut d'empilement en accord avec le calcul de Jouffrey et al. [10].

Les déplacements collectifs se produisent brutalement et créent des micromacles qui traversent la plage : ni le défaut $D$, ni la micromacle $A$ n'ont résisté au passage de la micromacle $\mathrm{EE}^{\prime}$. Le décalage en $F$ permet d'estimer à 700 environ le nombre des partielles qui sont passées. Ce micromaclage très hétérogène constitue l'essentiel de la déformation observée; les mouvements individuels, répartis de façon plus homogène, y contribuent peu.

3. Conclusion. - Ces deux exemples montrent que pour les teneurs du soluté considérées, les mécanismes qui régissent la déformation des alliages, ne sont pas l'extrapolation simple des mécanismes en jeu dans les alliages dilués. Qu'il s'agisse de nickel pur, d'alliage de nickel à moins de $15 \%$ de chrome, de cuivre, ou de cuivre-silicium à température assez basse, le mécanisme prépondérant était en effet le mécanisme de la traversée de la forêt.

L'énergie de défaut reste un critère important puisqu'elle entraîne la déformation par dislocations parfaites dans le $\mathrm{Ni}-33 \% \mathrm{Cr}$ et par dislocations dissociées ou par dislocations partielles dans le $\mathrm{Cu}$ $6,5 \% \mathrm{Si}$. Mais dans les deux cas considérés, le mode de déformation est essentiellement plan et l'on observe un mouvement collectif de dislocations entraînant une déformation très hétérogène. La force de frottement est élevée dans le $\mathrm{Ni}-\mathrm{Cr}$ à $0,19 T_{\mathrm{F}}$ où elle interdit plus ou moins le glissement dévié, alors qu'elle est très faible sur les partielles du $\mathrm{Cu}-\mathrm{Si}$ à $0,4 T_{\mathrm{F}}$ dont on observe directement le mouvement.

Ces observations in situ comme celles réalisées au microscope optique sur d'autres alliages [13] montrent l'importance des phénomènes de déformation hétérogène, de multiplication, et confortent certaines hypothèses [14] sur le mouvement collectif [15] des dislocations dans les alliages présentant un effet $\langle\Delta \sigma »$ (crochet après relaxation et reprise de la traction [9]) ou un effet Portevin-Le Chatelier importants. 


\section{Bibliographie}

[1] Nix, W. D. et Menezes, R. A., Ann. Rev. Mat. Sci. 1 (1971) 313.

[2] Friedel, J., Dislocations (Pergamon Press, London) 1964, 362.

[3] SuZUKI, H., Dislocations and Mechanical Properties of Crystals (J. Wiley, New York) 1957, 361.

[4] Clement, N. et Coulomb, P., Phil. Mag. 30 (1974) 663.

[5] Nordstrom, T. V. et Barrett, C. R., Acta Met. 17 (1969) 139.

[6] Clement, N. et Escribe, C., à paraître.

[7] Clement, N., Thèse de Doctorat (Toulouse) (1975).

[8] Clement, N., Monchoux, F. et Martin, J. L., Strength of metals and alloys (ICSMA 5) (Pergamon Press) 1979. 1299.
[9] Coujou, A., Phil. Mag. A 40 (1979) 173.

[10] Jouffrey, B., Daniel, A., EsCaig, B., J. Physique Colloq. 27 (1966), C3, 114.

[11] Mahajan, S. et Chin, G. Y., Acta Met. 22 (1974) 1113.

[12] Hirth, J. P., Metal. Trans. 1 (1970) 2367.

[13] Neuhauser, H., Schwink, Ch., Traub, H., Acta Met. 25 (1977) 1289.

[14] Korbel, A., Scripta Met. 8 (1974) 609.

[15] Martin, J. L., Revue Phys. Appl. à paraître, 1980. 